\title{
EDITORIAL
}

\section{„Neue Steuerung“ - Renaissance der Kybernetik?}

\section{"New Governance" - Renaissance of Cybernetics?}

Mit der Teilnahme Deutschlands an der Trends in International Mathematics and Science Study (TIMSS) im Jahr 1997, am Programme for International Student Assessment (PISA) der OECD ab dem Jahr 2000 und an der Progress in International Reading Literacy Study/Internationale Grundschul-Lese-Untersuchung (PIRLS/IGLU) und TIMSS im Grundschulbereich ab dem Jahr 2001 setzte ein Prozess ein, bei dem die bisherigen Steuerungsmodelle im Schulsystem einer kritischen Überprüfung unterzogen und in dessen Folge die bisherige Steuerung über den Input (z. B. über Lehrpläne) durch eine stärker am Output orientierte Steuerung ersetzt wurde. Diese „Neue Steuerung" war zudem unmittelbar anschlussfähig an bildungspolitische Entscheidungen und reformpädagogische Bemühungen in den 1990er-Jahren, den einzelnen Schulen eine größere Selbstverantwortung einzuräumen.

Elemente dieser "Neuen Steuerung“ sind die von der Kultusministerkonferenz (KMK) beschlossenen Bildungsstandards ebenso wie deren Überprüfung mittels repräsentativer Untersuchungen (IQB-Bildungstrends) und die Bereitstellung von Vergleichsarbeiten (VerA) durch das Institut zur Qualitätsentwicklung im Bildungswesen (IQB), eine indikatorengestützte Bildungsberichterstattung im Bund wie auch in vielen Ländern und Kommunen und die Einführung von Schulinspektionen. Die verschiedenen Elemente fasste die KMK im Jahr 2006 erstmals zu einer „Gesamtstrategie für das Bildungsmonitoring" zusammen. Eine Überarbeitung und Aktualisierung erfolgte im Jahr 2015. Gleichzeitig legte das Bundesministerium für Bildung und Forschung (BMBF) in seiner Zuständigkeit für die Forschungsförderung im Jahr 2007 das „Rahmenprogramm Empirische Bildungsforschung" mit dem Ziel auf, die Länder bei der schulischen Qualitätsentwicklung durch die Bereitstellung von mehr empirischem Wissen zu unterstützen.

Durch die verschiedenen, in der Gesamtstrategie der KMK zusammengefassten Monitoringmaßnahmen sollten die Verantwortlichen auf den verschiedenen Ebenen des Schulsystems Rückmeldungen zu den Ergebnissen ihrer Arbeit erhalten und diese zum Anlass nehmen, in ihren jeweiligen Zuständigkeitsbereichen qualitätssichernde und qualitätsentwickelnde Initiativen zu ergreifen. 
Kritiker*innen dieser neuen Steuerungsstrategie sehen dabei Analogien zu kybernetischen Steuerungsmodellen, die in Deutschland in den 1960er-Jahren (u. a. unter dem Stichwort „Programmiertes Lernen“) zwar diskutiert wurden, aber bald wieder in Vergessenheit gerieten. Nach ihrer Auffassung entsprechen - vereinfacht gesagt - die Kreisläufe aus „Datenerhebung und Rückmeldung“ der verschiedenen Monitoringmaßnahmen auf der Systemebene, auf der Schulebene und auf der Unterrichtsebene einfachen kybernetischen Steuerungsmodellen, die zudem zu einer möglichen Entpolitisierung von Steuerungsfragen beitrügen.

Im Zuge der in den vergangenen Jahren verstärkt einsetzenden Diskussion über eine stärkere Nutzung digitaler Lernumgebungen bekommen kybernetische Steuerungsvorstellungen eine zusätzliche Bedeutung auf der individuellen Ebene, wenn zum Beispiel in (einfachen) Lernprogrammen Aufgaben zu beantworten sind und die Schüler*innen unmittelbar ein Feedback zu ihren Leistungen bzw. zu ihrem Antwortverhalten bekommen. Aber auch jenseits dieses eher technischen Kontextes sind kybernetische Steuerungsvorstellungen in der schulischen Praxis verbreitet. So stellen sich u.a. Fragen, wie formative Assessments gestaltet sind bzw. gestaltet sein müssten, um (komplexen) individuellen Lernprozessen $\mathrm{zu}$ entsprechen, und welche Steuerungsvorstellungen und Menschenbilder dem immer stärker propagierten „selbstgesteuerten Lernen“ zu Grunde liegen.

Mit den aufkommenden „learning analytics“ wird zudem eine neue Qualität von Datenerfassung und (automatisierter) -auswertung erreicht. Daten zu Schul- bzw. Lernleistungen, zum Lernverhalten, zur Lerngeschwindigkeit, zu genutzten Medien und Informationsquellen, zu Aspekten von Selbststeuerung, Konzentration und Ausdauer usw. sollen nicht mehr nur punktuell durch (un-)regelmäßige Überprüfungen, Inspektionen oder Fragebögen erhoben werden, sondern vielmehr beiläufig und fortlaufend während der Interaktion der Schüler*innen mit digitalen Bildungsmedien. Diese Daten sollen nicht mehr nur in der Schule erfasst werden, sondern auch im privaten Bereich der Schüler*innen, wenn eigene Geräte zum Einsatz kommen. Mittels entsprechender Algorithmen sollen die Daten ausgewertet und an den bzw. die Schüler*in, aber auch an die Lehrkräfte zurückgemeldet und möglicherweise Dritten für Auswertungen und Profilbildungen zur Verfügung gestellt werden.

Mit dem vorliegenden Beiheft sind drei Ziele verbunden: Es soll erstens einen Beitrag zur Beantwortung der Frage leisten, ob auf den verschiedenen Steuerungsebenen eher technokratische Steuerungsauffassungen vorherrschen oder welche über kybernetische Kreisläufe hinausweisenden und stärker entwicklungsorientierten Modelle auf den verschiedenen Steuerungsebenen Anwendung finden - und mit welchen Folgen. Es soll zweitens durch die Darstellung von Positionen und Auffassungen zum Bildungsmonitoring und $\mathrm{zu}$ im Schulwesen empirisch vorzufindenden Steuerungspraktiken einen Beitrag zu einem übergreifenden Diskurs über Steuerungsfragen und Steuerungsmodelle im Schulsystem leisten. Schließlich soll es 
drittens mögliche Risiken und Folgewirkungen von zunehmenden Verdatungen des Pädagogischen durch „learning analytics“ aufzeigen und damit den Einstieg in eine in diversen Politik- und Technikbereichen bereits eingesetzte und selbstverständliche Technikfolgenabschätzung ermöglichen.

Die vorliegenden Beiträge sind das Ergebnis eines Calls der Redaktion der DDS aus dem Frühjahr 2019. Über die E-Mail-Verteiler verschiedener Fachgesellschaften wurden Wissenschaftler*innen gebeten, Abstracts zu möglichen Beiträgen für das Beiheft einzureichen. Die Herausgeberin und die beiden Herausgeber des Beiheftes sowie die Redaktion der DDS haben die eingegangenen Abstracts mit Blick auf die Zielsetzung des Beiheftes gesichtet und insgesamt elf „angenommen“, d.h., sie haben die Wissenschaftler ${ }^{*}$ innen, die die Abstracts eingereicht hatten, um ein entsprechendes Manuskript gebeten.

Die eingegangenen Manuskripte wurden zunächst von der Herausgeberin und den beiden Herausgebern des Beiheftes sowie von der Redaktion der DDS geprüft und anschließend von jeweils zwei externen Gutachter*innen in einem Double-Blind-PeerReview-Verfahren begutachtet. Zwei der ursprünglich geplanten Beiträge sind aus unterschiedlichen Gründen dann leider doch nicht zustande gekommen.

Die Beiträge sind von uns in Kapiteln zusammengefasst worden, die den verschiedenen Ebenen des Schulsystems folgen. Zwei Beiträge behandeln Aspekte des Systemmonitorings, drei einzelschulische Steuerungsfragen und drei Aspekte von „Verdatungen“ des Pädagogischen, also vorrangig die Ebene des Unterrichts.

Eingeleitet wird das Beiheft unter der Kapitelüberschrift „Ansätze einer Theorie zur ,Neuen Steuerung“ mit dem Beitrag „Neue Steuerung' im Schulsystem als kybernetisches Steuerungsmodell?" von Nils Berkemeyer. Er vertritt darin die Auffassung, die Forschung zur Steuerung im Schulsystem sei nach wie vor defizitär, insbesondere in Bezug auf Theoriebildung und -nutzung. Es stünden sich zwei Gruppen gegenüber: jene Bildungsforscher*innen, die behaupten, Evidenz zu erzeugen und durch Wissen und unter Verzicht auf normative Diskurse einen Beitrag zur Steuerung leisten zu können, und jene Vertreter*innen der von ihm so genannten „Governance-Pädagogik“, die mit dem Monitoring verbundene Machtfragen und den technologischen bzw. kybernetischen Charakter der Neuen Steuerung kritisieren. Beide Positionen sind - so seine These - wenig hilfreich in Bezug auf Steuerungsfragen, weil sie Evidenz und Moral als Alternativen behandelten. Er bescheinigt beiden eine Abstinenz von an Steuerungstheorien orientierten Reflexionen.

Doch gerade in Bezug auf die „Neue Steuerung“, die als evidenz- und datenbasierte Steuerung auf Wissen setzt, lassen sich seines Erachtens klassische Steuerungstheorien der politischen Kybernetik sowie systemtheoretische Weiterentwicklungen nutzen, um wesentliche Befunde zur „Neuen Steuerung“ vergleichsweise umfassend zu erklären. 
Das Bildungsmonitoring habe seines Erachtens kaum zu Struktur- und Organisationsveränderungen bei den Einzelschulen geführt. Zudem führten die andauernden schulischen Rückmeldungen zu Überforderungen von Schulen. Durch eine Kopplung der Schulen an Wissenschaft und Bildungsadministration könnten im Prinzip neue Entscheidungsroutinen entstehen und die Entscheidungen in Schule beschleunigen und rationaler machen. Ernsthafte Kopplungsversuche mit der Einzelschule fänden jedoch kaum statt. Berkemeyer vertritt ferner die Auffassung, dass es mit Datenerfassung und -rückmeldung, Zielfindung und -formulierung, Umsetzungsphase, erneuter Überprüfung als Elementen der „Neuen Steuerung“ vorrangig nicht um eine klare Zielsteuerung gehe, die man in einem kybernetischen Programm erwarten würde. Es gehe stattdessen um ein Legitimationsprogramm der klassischen Input-Steuerung, das die „Vorgabenpolitik“ nicht kritisch in den Blick nehme, sondern Verbesserung im Rahmen der bestehenden Vorgaben annehme. So müsse „Neue Steuerung“ seines Erachtens dann doch eher als „neue Schulentwicklung“ im Verantwortungsbereich der Einzelschule verstanden werden.

Zusammenfassend hält er fest, dass kybernetische Perspektiven auf Steuerungsprozesse im Schulsystem wertvoll seien und ihr analytisches Potenzial längst nicht ausgeschöpft worden sei. Im Vordergrund stehe allzu häufig vorschnelle Kritik. Zugleich hätten kybernetische Ansätze aber auch Grenzen, wie alle anderen Ansätze auch; darum seien sie zu ergänzen oder im Zuge von Theorie-Theorie- oder Theorie-EmpirieTriangulationen $\mathrm{zu}$ überprüfen und weiterzuentwickeln. Das Lern- und Analysepotenzial durch den Transfer steuerungstheoretischer Arbeiten sei seines Erachtens allemal höher als überkritische Kommentierungen der „Neuen Steuerung“ und unter Verweis auf Governance stattfindende Fallbeschreibungen.

Im Anschluss an diese eher grundsätzlichen Überlegungen betrachten Annina Förschler \& Sigrid Hartong im ersten Beitrag des nachfolgenden Kapitels „Monitoringsysteme“ exemplarisch „Datenpraktiken des Schulmonitorings in staatlichen Bildungsbehörden“.

Sie berichten erste Ergebnisse eines laufenden DFG-Projektes, das anhand von Fallstudien auf der Ebene von Bundesländern in Deutschland und Bundesstaaten in den USA die zunehmende Expansion und Zentralisierung digitaler Schulmonitoringsysteme (z. B. durch die Einrichtung zentraler Schulverwaltungssoftware, die Zusammenführung unterschiedlicher Leistungsdaten usw.) untersucht. Mit dem Projekt gehen sie der Frage nach, inwieweit bestimmte Steuerungsvorstellungen, -modelle und -praktiken eine zunehmende Datafizierung und Digitalisierung im Bildungssektor auf den verschiedenen Steuerungsebenen fördern. Gleichzeitig untersuchen sie, inwieweit sich lokale bzw. kulturell-institutionelle Kontexte in den Praktiken der Datenproduktion manifestieren. 
Methodisch basieren ihre Falluntersuchungen zum einen auf der Analyse von Dokumenten, die Organigramme, Tätigkeitsberichte, Beschreibungen von und Anleitungen zur Nutzung von einzelnen Monitoring-Instrumenten bzw. digitalen Datenplattformen, Produktportfolios von Softwareherstellern oder Pressematerialien für jedes Bundesland bzw. jeden Bundesstaat umfassen, und zum anderen auf 39 leitfadengestützte, narrative Interviews mit Mitarbeiter*innen der Schulbehörden, aber auch von Qualitätsinstituten sowie IT-Organisationen in zwei deutschen Bundesländern und zwei amerikanischen Bundesstaaten. Übergeordnetes Ziel des Projektes ist es, mehr über das Wie der jeweiligen Datenproduktion, -verarbeitung und -nutzung zu erfahren.

Die vorgestellten Ergebnisse auf der Ebene der Bundesländer zeigen, dass den Schulmonitoringsystemen oftmals simple Regelkreismodelle zugrunde liegen, es sich in der Praxis aber um ein hochkomplexes Zusammenspiel unterschiedlicher, oftmals ambivalenter Logiken und Einflüsse handelt, die wiederum jeweils spezifische Potenziale und Problematiken mit sich bringen, die den Behördenmitarbeiterinnen zum großen Teil auch sehr bewusst sind. Im Beitrag wird dies anhand von ausgewählten Beispielen diskutiert. Dabei wird gleichzeitig sichtbar, wo und wie sich Gemeinsamkeiten und Unterschiede zwischen Deutschland und den USA manifestieren. Insbesondere die Nutzung von Daten für Accountability-Zwecke oder die Veröffentlichung von Daten werden in Deutschland weitaus skeptischer beurteilt als in den USA.

Deutlich wird auch die hochgradig politische Seite von Schulmonitoring und Dateninfrastrukturen, die in einfachen Regelkreismodellen oftmals unterbelichtet bleibt. Dateninfrastrukturen sind - so die beiden Autorinnen - immer wertbehaftete Instrumente der Selektion. Ihre Ergebnisse zeigen, dass Dateninfrastrukturen nicht nur neue Kategorien schaffen (z. B. Schulen mit einem bestimmten Sozialindex oder, wie im Fall der USA, schools (not) meeting targets), sondern letztendlich auch die Bildungswelt selbst verändern, indem sie Dinge auf besondere Weise (un)sicht-, wahrnehm-, benenn- und damit steuerbar machen. Sie plädieren folglich dafür, Forschung im Bereich der Critical Data Studies auszubauen sowie einen Wissenstransfer zwischen deren Erkenntnissen und der täglichen Praxis von Datenproduzent*innen, Datenmanager*innen, IT-Expert*innen, aber auch Schulleitungen und Lehrkräften zu fördern, um ein kritisches Bewusstsein für die Komplexität der Datenproduktion und für die fehlende Neutralität von Daten und Monitoring-Instrumenten zu schaffen.

Britta Klopsch \& Anne Sliwka zeigen am Beispiel der kanadischen Provinz Alberta, wie verschiedene Daten(quellen) in einem Schulsystem so genutzt werden können, dass sie über unterschiedliche Ebenen hinweg dazu beitragen, das Lernen und die Leistungen der Schüler*innen zu verbessern. Das Lernen der Schüler*innen werde mehrdimensional erfasst und wahrgenommen, und die Vielfalt der erhobenen Daten erlaube es, die Komplexität des Systems Schule differenziert abzubilden. 
Jede Art von Daten, die zu einer Verbesserung des Lernens bei Schüler*innen führen solle, müsse interpretiert werden. In der Provinz Alberta erfolge eine reflektierte Auseinandersetzung mit den Daten in einem deliberativen Prozess auf allen Ebenen des Schulsystems, von der einzelnen Lehrkraft, der Schulleitung, der Schulaufsicht bis hin zum Bildungsminister bzw. der Bildungsministerin. Die jährlich in einem festgelegten Zyklus erhobenen, digital aufbereiteten und zielgruppenspezifisch zur Verfügung gestellten Daten informierten darüber, wo die einzelnen Schulen und das ganze Schulsystem stünden, und seien Ansatzpunkt von regelhaft stattfindenden strategischen Gesprächen und daraus abgeleiteten gemeinsamen Handlungsschritten auf den verschiedenen Ebenen des Schulsystems:

Lehrkräfte und Schulleitungen prüften in Abstimmung mit speziellen (Förder-)Fachkräften stetig datengestützt, in welchen Bereichen die Schüler*innen Unterstützung benötigten, um noch erfolgreicher lernen zu können. Im Zusammenhang damit stehe auch die Frage, welches Wissen die Lehrenden benötigen, um die Schülerinnen in ihrem Lernen optimal zu unterstützen.

Die Dateninterpretation sei nicht alleinige Aufgabe der Schulen. Alle Ebenen des Systems fühlten sich für die Erreichung der drei strategischen Ziele der Provinz Leistung, Chancengerechtigkeit und Wohlbefinden - gleichermaßen verantwortlich und arbeiteten dafür eng zusammen. Alberta gelinge auf diese Weise sowohl eine horizontale als auch - durch Zielvereinbarungen zwischen Ministerium, Schulaufsicht und Schulleitungen - eine vertikale Verknüpfung.

Durch die enge Verschränkung von Datennutzung und deliberativer Dateninterpretation in professionellen Lerngemeinschaften zeige die datengestützte Schulentwicklung in der kanadischen Provinz Alberta, dass es möglich ist, gelingende Entwicklungen zum Wohle der Schüler*innen zu initiieren. Gelingensbedingung dabei sei, keine spezifischen „Verfahrensvorschriften“ zu erarbeiten, die quasi automatisch die gegebenen Ziele erreichen sollen, sondern die gewonnenen Daten mit den Beobachtungen und Wahrnehmungen der professionellen Akteure so zusammenzubringen, dass in der Gesamtschau handlungsleitende Strategien zur Verbesserung des Schülerinnenlernens entstehen. Dies gehe - so die beiden Autorinnen - über das traditionelle Verständnis von Kybernetik hinaus, weil es die Stärke systematischer Datennutzung mit der Stärke menschlicher Kooperation zum Nutzen der Lernenden in Beziehung setze.

Im ersten Beitrag des Kapitels „Die Ebene der Einzelschule“ werden von Melanie Schmidt zwei mögliche Herkünfte der Steuerungslogik von Schulinspektionen als Instrumenten einer neuen Steuerung im Schulsystem diskutiert. An der Vorstellung von Schulentwicklung durch Einsicht als zentralem Wirkmechanismus von Schulinspektionen lassen sich ihres Erachtens die Vollzugslogiken der „Neuen Steuerung“ exemplarisch verdeutlichen. Verbunden mit der Absicht einer Reflexion 
des Konzepts der „Einsicht“ zeigt sie auf, dass Schulinspektionen die Forderung schulischer Autonomie - als eigenverantwortlich vorzunehmender einzelschulischer Selbststeuerung - unterstützen, indem sie Daten über Schulen sammeln und diese anschließend rückmelden. Zugleich sei aber mit Schulinspektionen eine Tendenz zur Homogenisierung und Nivellierung von Differenzen und damit zur individualisierten Anpassung an von außen gesetzte Vorgaben verbunden. In dieser Hinsicht zeigten sich Nähen zur Kybernetik, die von den auf Datenrückmeldung bezogenen Selbstregulationsmöglichkeiten eines autonomen Systems ausgeht. Zu dem Konzept der „Einsicht“ finden sich ihres Erachtens zudem auch in pädagogischen Kontexten Überlegungen zur Verbindung von Wissen und Regulierungshandeln - etwa in der Rousseauschen „negativen Erziehung“. Entlang der beiden Programm- bzw. Theorielinien (Kybernetik und "negative Erziehung") exploriert sie, inwiefern Schulinspektionen als Steuerungsinstrumente an diese beiden unterschiedlichen Diskursstränge anzuknüpfen vermögen. Gemäß dem Ansinnen einer reflexiven Reformfolgenforschung fragt sie nach dem "Neuen“ in der "Neuen Steuerung" von Schulen und kommt abschließend $\mathrm{zu}$ dem Ergebnis, dass mittels Schulinspektionen eine Angleichung von Schule an politisch-administrative Logiken erfolge. Diese Angleichung unterlaufe jedoch das grundlegende Ansinnen der „Neuen Steuerung“, das sich auf Qualitätsentwicklung beziehe. Qualitative Veränderungen und neue oder erweiterte Handlungs- bzw. Gestaltungsspielräume formierten sich aber erst im Verhältnis einer Differenz zum Bestehenden.

Daniel Goldmann befasst sich im zweiten Beitrag dieses Kapitels mit der Frage der Steuerung von evolutionären Veränderungen. Dabei geht er von einem fortwährenden Auseinanderfallen von Reform- und Steuerungserwartungen auf der einen und der Realität der schulischen Praxis auf der anderen Seite aus, das sich in Studien zu den Wirkungen „Neuer Steuerung“ zeige. Zwar seien die Reformen keineswegs folgenlos gewesen und hätten durchaus auch intendierte positive Wirkungen erzielt. Mehrheitlich seien die Effekte jedoch entweder in der Summe eher negativ und ambivalent oder zeigten kaum die beabsichtigten Folgen für Unterricht und Schule. So nutzten beispielsweise Lehrkräfte Daten aus den Vergleichsarbeiten vornehmlich zur Bestätigung der eigenen unterrichtlichen Praxis und ihrer Selektionsentscheidungen, aber nur selten zur Diagnostik oder kritischen Selbstreflexion und noch seltener als Grundlage für eine datengestützte Schul- und Unterrichtsentwicklung mit dem gesamten Kollegium. Ebenso sei die Akzeptanz der Schulinspektion in den Schulen hoch; die intendierten Impulse für eine Weiterentwicklung von Schule und Unterricht oder gar die Steigerung der Leistungen von Schüler*innen hätten bisher aber nicht nachgewiesen werden können.

Die Educational Governance sei mit dem Anspruch gestartet, traditionelle und all$\mathrm{zu}$ simple Steuerungsvorstellungen und damit auch allzu simple Reformhoffnungen hinter sich zu lassen, um so das Auseinanderklaffen von Hoffnung und realer Praxis besser verstehen zu können. Dafür seien neue Begriffe wie Handlungskoordination, 
Mehrebenensystem u. a. eingeführt worden, die die Prozesse im Bildungssystem komplexer abbilden sollen. Aber auch dieser Anspruch sei bisher nicht umfassend eingelöst worden. Nach wie vor würden v. a. in Verwandtschaft mit einer Kybernetik erster Ordnung stehende zweckrationale Handlungstheorien verwendet, um verwertbares Steuerungswissen zu generieren. Mittels der Luhmannschen Systemtheorie vollzieht der Autor in seinem Beitrag einen Wechsel von der Kybernetik erster zu einer Kybernetik zweiter Ordnung und wendet damit anstelle zweckrationaler Theorien eine komplexere Sozialtheorie an. Diesen Wechsel stellt er sowohl in seiner theoretischen Grundlegung als auch in der Beschreibung einzelschulischer Entwicklung vor. Anschließend rekonstruiert er als zentrale Problemlage schulischer Entwicklung, dass Schulen in ihrer organisationalen Verfasstheit nur unzureichend aufgestellt seien, um interne Meinungsverschiedenheiten bzw. Entscheidungskonflikte - als zentralen Motor für Veränderungen - produktiv austragen zu können. Auf Basis empirischer Ergebnisse skizziert er dann, wie Schulen trotz dieser schwierigen, von ihm als paradox bezeichneten Ausgangslage Schulentwicklung betreiben können. Abschließend diskutiert er, welche Anregungen eine solche theoretische Neujustierung für die einzelschulische Entwicklungsarbeit und die politisch-administrative Steuerung von Schulentwicklung bereithält. Sie sei u. a. mit einer Einladung an Akteure der politischadministrativen Steuerung verbunden, sich selbst in spezifischen Problemlagen und bei Handlungsherausforderungen als Akteur im Schulsystem besser zu verstehen.

Stefan Hahn, Stanislav Ivanov \& Roumiana Nikolova berichten in ihrem Beitrag „Innovation und Transfer brauchen mehr als Daten zum Output“ über Erkenntnisse aus begleiteten internen Evaluationen. Vorgestellt werden von ihnen die Anlage und die Unterstützungsstrukturen der in Hamburg angebotenen schulinternen Evaluation mit dem längsschnittlich normierten Instrumentarium der Schulleistungsstudie Kompetenzen und Einstellungen von Schülerinnen und Schülern (KESS). Anhand ihrer Erfahrungen zeigen sie exemplarisch auf, in welcher Variationsbreite KESS von Einzelschulen zu Zwecken der eigenverantwortlichen Schul- und Unterrichtsentwicklung genutzt wird. Die Erhebung primärer Daten mit dem KESS-Instrumentarium ermögliche es, viele schulspezifische Fragestellungen in vielfältigen Evaluationsdesigns zu bearbeiten. Die schulischen Akteure folgten bei der Datennutzung häufig der kybernetischen Vorstellung einer funktionalen Optimierung bestehender Routinen. Ihnen werde durch ein spezifisches Feedback, das die Rückmeldung von Kompetenz- und Einstellungsdaten von Schülerinnen mit einer gemeinsamen Interpretation dieser Ergebnisse verknüpft, die Möglichkeit einer grundlegenden Veränderung der Prozessmuster eröffnet. Insbesondere die im Zuge dieser Interpretation erfolgende Kontextualisierung von Daten zum Output mit qualitativen Prozessdaten und dem Erfahrungswissen von Lehrkräften markiere den Kern einer wissenschaftlichen Begleitung von Prozessen des Transfers pädagogischer Prototypen im Sinne einer designbasierten Schulentwicklung. 
„Neue Steuerung“ - Renaissance der Kybernetik? |

Die Beiträge des letzten Kapitels fokussieren unter der Überschrift „,Verdatung des Pädagogischen?" auf die Ebene des Unterrichts bzw. auf das Lernen der Schüler*innen und das professionelle Lehren der Lehrkräfte.

Zunächst wirft Anke Redecker unter der Überschrift „Von der Selbststeuerung zur interaktiven Irritation“ einen kritischen Blick auf schulische Kontrollsubjekte. Dafür problematisiert sie zunächst Selbstregulierungsprozesse im schulischen Kontext vor dem Hintergrund kybernetischer Steuerung, um vermeintliche Autonomiegewinne der Steuernden und Gesteuerten zu hinterfragen. Hieran anschließend diskutiert sie in einem weiteren Schritt eine Kybernetik-kritische Bildung, die unhinterfragte Regelkreise in schulischen Kontrollprozessen zu durchbrechen lernt und sich hierzu wieder auf die Anregung von Lernprozessen durch einen (pädagogisch professionellen) Anderen besinnt.

Wenn selbstgesteuerte Lehr-Lern-Prozesse den Unterricht beherrschen, steht ihres Erachtens nicht mehr Bildung im Fokus, sondern Ausbildung, nicht mehr die Anregung Lernender, sich verstehend und kritisch $\mathrm{zu}$ anderen, anderem und sich selbst ins Verhältnis zu setzen, sondern ein berufstauglicher Lernertrag und effiziente Lernleistungen, deren Messung aufzeigen soll, inwieweit ein selbst geplanter, aber letztlich weitgehend fremdbestimmt geregelter Lernprozess erfolgreich war. Wenn die Planung hierfür nicht gut genug war, könne bzw. müsse man zukünftig besser planen.

Was aber, wenn gerade das Nichtplanbare, das auf die Grenzen des Planens aufmerksam macht, für lernförderliche Überraschungen sorgt, die sich auch zukünftig in kein Planungsschema pressen lassen? fragt sie. Dann komme es ihrer Ansicht nach darauf an, Regelkreise nicht immer wieder neu auszurichten, sondern zu durchbrechen und dabei zugleich zuzugestehen, dass sich menschliches Handeln nicht in Steuerung erschöpft. Es komme also darauf an, die eigenen widerständigen Potenziale letztlich nicht nur zu aktivieren, sondern zugleich überlegt und verantwortlich einzuschätzen und einzusetzen. Dies aber erfordere ein Umlernen in Bezug auf den bisherigen kybernetischen Kurs, zumal dieses Umlernen einen Bruch mit der Ideologie der Planund Machbarkeit bedeuten würde, denn Lernprozesse könnten nicht nur auf ungeplantes, sondern oft auch auf unplanbares Neuland führen.

Kritische Bildung basiere auf krisenhaften Prozessen des Umlernens, die einer irritierenden Intervention durch Lehrende bedürften. Vor allem Lehrende müssten deshalb Lernformen und -methoden auf ihr Bildungspotenzial hin hinterfragen, und es sei ihre Aufgabe zu entscheiden, welche Art des Lernens in einer konkreten Unterrichtssituation sinnvoll eingesetzt werden könne.

Markus Reinisch geht dann in seinem Beitrag „Big Data und Algorithmen: Instrumente einer neuen kybernetischen Steuerung an Schulen?“ der Frage nach, inwieweit die vielfach gesellschaftlich erprobten Vermessungstechniken als „learning analytics“ und 
als Instrumente einer neuen kybernetischen Steuerung an Schulen gesehen werden können.

Er beschreibt, dass quer durch alle Schularten und Bundesländer im Rahmen bildungspolitischer Offensiven neue Medienkonzepte erstellt und in Strategiepapieren Vorschläge für eine umfassende Verdatung unterrichtlicher Prozesse gemacht werden. Dazu sollen u.a. „learning analytics“ weiterentwickelt und im Sinne von „smart devices" systematisch im Unterricht eingesetzt werden, denn neue Erkenntnismöglichkeiten seien die Voraussetzung, um auf die Herausforderungen in der digital geprägten und globalisierten Welt vorbereitet zu sein und im internationalen Vergleich nicht zurückzubleiben, wie Protagonist*innen von „learning analytics“ behaupten.

Die viel diskutierte Outputorientierung im Rahmen der „Neuen Steuerung“ soll den Strategiepapieren zufolge "data-driven“ in der Schule, im Unterricht und bei den individuellen Lernprozessen fortgesetzt werden - mit möglicherweise weitreichenden Konsequenzen, nicht nur auf der institutionellen, sondern auch der individuellen Ebene für den Erziehungs-, Bildungs- und Subjektwerdungsprozess der Heranwachsenden. Algorithmenbasierte Auswertungsmöglichkeiten der bei der Nutzung von Bildungsmedien erhobenen Daten und die dadurch potenziell möglichen Eingriffe in Lern- und Bildungsbiografien stellen seines Erachtens in ihrer Gesamtheit eine Form neuer kybernetischer Steuerung dar.

Der Autor stellt in seinem Beitrag vier Szenarien vor, in denen gefordert wird, dass der zu erwartende Fortschritt bei den digitalen Bildungswelten auch in den Schulen zur Anwendung kommen müsse. Dabei stellt er die Nähe der Vorschläge zur pädagogischen Kybernetik der 1960er-Jahre kritisch heraus, um anschließend einige mögliche Folgen für das lernende Individuum zu beleuchten. Mit den ausgewählten Szenarien und einem zusätzlichen Blick in bildungspolitische Strategiepapiere will er verdeutlichen, inwiefern ein zuvor von ihm beschriebener Nexus von Ökonomie, Mathematisierung und Digitalisierung auch den medienpädagogischen Diskurs beeinflusst (hat).

Die skizzierten Szenarien rund um ein kybernetisches, kennziffern- und sollwertgeleitetes Steuern werfen für ihn eine Reihe von Fragen auf, die allesamt noch nicht annährend beantwortet werden können. Wie steht es um den Datenschutz, wenn IT-Konzerne den Schulen Clouds zur Verfügung stellen, in denen die Schüler*innenprofile gespeichert sind, die nicht nur auf rein schulische Prozesse bezogene Daten enthalten? Was geschieht mit Formen der Leistungserhebung, die digital nicht erfasst werden können, weil sie komplexer sind als ein geschlossener Regelkreis, z.B. bei Aufgaben zur Interpretation eines Sachverhalts oder freieren Aufgabenformen, die Assoziationen einfordern oder das Vertreten und Begründen eines eigenen Standpunktes? Wer erstellt die "Lernmodule“ und die Inhalte der „Lernpfade“? Wird es in dieser Hinsicht eine noch stärkere Zusammenarbeit zwischen 
Wirtschaft und Schulen geben, sodass die Ökonomisierung von Bildung unter digitalen Vorzeichen weiter voranschreitet? Wie (demokratisch) erfolgt das Setzen von Sollwerten? Inwieweit fließen algorithmen-ethische Aspekte in die Szenarien ein? Wer bestimmt, wie stark die Handlungsmacht der Algorithmen sein wird?

Vor allem wird seines Erachtens aber die Frage von Belang sein, wer was als Störgrößen außerhalb des kybernetischen Systems definiert. Zufälle und Überraschungen würden durch determinierende und algorithmisch-definierende kybernetische Vorgaben eingeschränkt. Es bleibe wohl (zu) wenig Raum für Irritationen und Perturbationen, um beispielsweise verschiedene Sichtweisen auf einen Sachverhalt zuzulassen.

Martin Karcher geht bei seinem mit „Die (kybernetische) Bändigung des Zufalls Dataveillance und Learning Analytics als Herausforderung erziehungswissenschaftlicher Reflexion" überschriebenen "Versuch einer Technikfolgenabschätzung" aus erziehungswissenschaftlicher bzw. grundlagentheoretischer Perspektive den „pädagogischen Annahmen" und den möglicherweise daraus folgenden praxisinhärenten Normen von „learning analytics" nach. Es könne sich durch einen breiten Einsatz von „learning analytics“ eine neue Form von kontinuierlichen (Über-)Prüfungen etablieren, die Einfluss auf die pädagogische Praxis nehme und damit eine Reihe von erziehungswissenschaftlichen Fragen aufwerfe. Im Einzelnen diskutiert er mit Bezug auf Foucaults Prüfungsbegriff das Konzept der Dataveillance, d.h. das Konzept einer unsichtbaren digitalen Überwachung, und zeigt, inwiefern „learning analytics“ dieser neuen permanenten Prüfungslogik entsprechen. Im Anschluss daran diskutiert er drei Dimensionen tradierter erziehungswissenschaftlicher Theoriebildung im Zusammenhang mit „learning analytics“: Bildsamkeit und das „Versprechen der Bildung", das Technologiedefizit der Erziehung und schließlich pädagogische Professionalität. Erkenntnisleitend ist dabei für ihn jeweils die Frage, zu welchen Irritationen und Verschiebungen es durch eine breite Implementation von „learning analytics" kommen könnte und vielleicht auch schon kommt.

Neben der Erfassung und Auswertung vielfältigster Daten zu Lernprozessen von Schüler*innen haben „learning analytics“ aber auch noch zusätzlich einen prädiktiven Anspruch. Ihr Ziel ist auch eine verbesserte Steuerung pädagogischer Praxis durch den Versuch einer „probabilistischen Bändigung des Zufalls“. Karcher zeigt, dass im Zuge dessen drei Linien pädagogischer Theoriebildung mit neuen Fragestellungen konfrontiert sind: Das Versprechen der Bildung steht einer algorithmischen Vorhersehbarkeit entgegen, pädagogische Professionalität droht durch technisches Expertinnenwissen überformt zu werden, und das Technologiedefizit der Erziehung löst sich in Accountability auf. Solche Engführungen seien eine Herausforderung einer theorieorientierten Bildungsforschung und im Rahmen einer elaborierten Technikfolgenabschätzung zu problematisieren. 
Ein kurzes und vorläufiges Resümee der Beiträge des Beiheftes macht unseres Erachtens sechs Punkte deutlich:

- Das Konzept der „Neuen Steuerung“ bedarf einer stärkeren theoretischen Fundierung und empirischen Überprüfung seiner Wirksamkeit, wenn es nicht nur um Rechenschaftslegung, sondern auch um eine wissenschaftlich fundierte Verbesserung der Qualität von Schule und Unterricht gehen soll.

- Für eine solche Qualitätsverbesserung ist neben einer engen Kooperation der professionellen Akteure auf den verschiedenen Ebenen die Einbeziehung der Beobachtungen und Wahrnehmungen der Akteure vor Ort bei der Interpretation und Diskussion der Ergebnisse von Monitoring-Verfahren und der Ableitung von nächsten Handlungsschritten erforderlich.

- Dabei ist es im Sinne einer Kybernetik zweiter Ordnung notwendig, dass sich die politisch-administrativen "Steuerleute“ selbst als beobachterabhängige Akteure in spezifischen Problemlagen und Handlungsherausforderungen wahrnehmen und sich somit selbst als Akteure im Schulsystem verstehen.

- Schulmonitoring insgesamt sowie die eingesetzten Instrumente und Verfahren sind hochgradig politisch. Notwendig ist es deshalb, ein kritisches Bewusstsein für die Komplexität der Datenproduktion, für die Normgebundenheit und damit für die fehlende Neutralität von Daten und Monitoring-Instrumenten sowohl in der Bildungspolitik als auch in der Bildungsadministration, in den Schulen und insbesondere auch bei den Datenerheber*innen bzw. -produzent*innen in den Landesinstituten und Qualitätseinrichtungen und ggf. beauftragten externen wissenschaftlichen Einrichtungen zu schaffen.

- Die zunehmende „Datafizierung“ hat Folgen für die Gestaltung von Schule und Unterricht. Dringend erforderlich ist es, die „Risken und Nebenwirkungen“ interdisziplinär, d. h. sowohl von den Datenproduzent ${ }^{*}$ innen her als auch aus einer bildungstheoretischen Perspektive, in den Blick zu nehmen. Es geht dabei nicht um vorschnelle Kritik und Ablehnung der Instrumente und Verfahren bzw. um mögliche Rechtfertigungen, sondern um einen Diskurs über ein gemeinsames Ziel.

- Dies gilt insbesondere für eine breite Einführung und Nutzung von „learning analytics“. Die mit diesem Instrument verbundenen „Risiken und Nebenwirkungen“ für die Gestaltung des Unterrichts und des Lernens übersteigen die Herausforderungen für die Gestaltung des Unterrichts durch Monitoringsysteme bei weitem. Dabei geht es nicht nur um Datenschutzfragen, sondern vor allem um eine grundsätzliche Neubestimmung des Verhältnisses von Lernenden und Lehrenden mit weitreichenden Konsequenzen für das Konzept des selbstgesteuerten Lernens und das Verständnis von Professionalität und professionellem Handeln. 
- Kybernetik ist der Mantel für die Beiträge des Beiheftes. Sie hat unseres Erachtens das Potenzial zur besseren Bewältigung aktueller Steuerungsherausforderungen und kann zudem Perspektiven für einen theoretisch reflektierten Diskurs bieten, ohne die Komplexität, die dem Gegenstand „Steuerung von Schule“ innewohnt, zu simplifizieren.

Wir wollen das Editorial mit einem Lesehinweis abschließen: Im Jahr 1954 (!) veröffentlichte Isaak Asimov die Kurzgeschichte „Die Schule“ (Originaltitel: The Fun They Had), ${ }^{1}$ in der zwei Kinder, Margie und Tommy, zunächst über ein von Tommy auf dem Speicher gefundenes uraltes Buch sprechen. Tommy zeigt Margie das Buch am 17. Mai 2157. Beschrieben ist in dem Buch, wie Kinder früher in der Schule gemeinsam lernten und dabei von Lehrer*innen angeleitet wurden. Beide kennen keine Schule, sondern nur eine Bildschirmmaschine, einen „mechanischen Lehrer“, der in einem kleinen Nebenraum neben dem Kinderzimmer untergebracht ist und vor bzw. mit dem sie allein lernen. Der „mechanische Lehrer“ ist genau auf die Fähigkeiten des einzelnen Kindes eingestellt; dabei passieren auch Fehler. Margie liest das von Tommy gefundene Buch. Während sie ihre Hausaufgaben erledigt, stellt sie sich vor, wie es sein müsste, mit vielen Kindern zusammen in der Schule zu spielen, zu lachen und in einem großen Raum zusammen zu lernen und sich gegenseitig helfen zu können: „She was thinking about the fun they had“.

Die Kurzgeschichte sollte unseres Erachtens Anlass genug sein, vor einer breiten Einführung und Nutzung von „learning analytics“ kritisch über mögliche Konsequenzen nachzudenken. In vielen Politik- und Technikfeldern sind Technikfolgenabschätzungen ein bewährtes Instrument, um sich über mögliche „Risiken und Nebenwirkungen" vor der Einführung eines Programms oder einer technischen Neuerung ein Bild zu verschaffen. Wir halten eine solche Technikfolgeabschätzung im Zusammenhang mit „learning analytics“ für dringend erforderlich, und sie sollte von den politisch Verantwortlichen umgehend in Auftrag geben werden.

\section{Detlef Fickermann, Veronika Manitius \& Martin Karcher}

https://doi.org/10.31244/9783830991618.01

1 Die Kurzgeschichte ist in dem bei Heyne 2018 veröffentlichten Sammelband Isaak Asimov: Geliebter Roboter erschienen. Sie ist in mehreren Sammlungen von Kurzgeschichten für den Unterricht enthalten; man findet sie auch im Internet. 